\title{
Fachfremde Leistungen
}

\section{Fallstricke bei Arzthaftung und Außendarstellung beachten}

\author{
Ärzte dürfen im Bereich der privatärztlichen Leistungen und der Selbstzahlerleistungen \\ in gewissen Grenzen auch außerhalb ihres Fachgebietes tätig werden. Dies hat kürzlich das \\ Bundesverfassungsgericht (BVerG) entschieden.
}

$\mathrm{M}$ it seiner Entscheidung hat das BVerG seinen fast 40 Jahre alten „Facharztbeschluss“ weiterentwickelt und übermäßig restriktiven Tendenzen der Berufsordnungen eine Absage erteilt (Az.: 1 BvR 2383/10). Seit dem Facharztbeschluss von 1972 haben die Ärztekammern die Auffassung vertreten, jeder Arzt, der eine weiterbildungsrechtliche Gebietsbezeichnung führt, müsse sich bei seiner Behandlungstätigkeit auf dieses $\mathrm{Ge}$ biet beschränken. Ausnahmen seien nur in der Notfallbehandlung und dann zulässig, wenn der Patient aufgrund eines gewachsenen Vertrauensverhältnisses ausdrücklich eine fachfremde Behandlung wünsche.

Unter Berufung auf diese Rechtsprechung haben die ärztlichen Berufsgerichte in der Vergangenheit etwa einem Gynäkologen die Schmerztherapie bei einem Mann untersagt, einem Kinderarzt wurde die Behandlung Erwachsener verboten.

\section{Voraussetzung: Facharztniveau}

Die Verfassungsrichter in Karlsruhe haben nun klargestellt, dass fachgebietsfremde Leistungen berufsrechtlich dann zulässig sind, wenn sie von dem Arzt nur in geringfügigem Umfang erbracht werden. Dessen Obergrenze wurde freilich nicht definiert. Das Gericht hat jedoch festgestellt, dass ein Leistungsumfang von weniger als $5 \%$ eindeutig geringfügig sei. Als Bezugsgröße hat das Bundesverfassungsgericht im entschiedenen Fall die Gesamtzahl der von dem Arzt durchgeführten Operationen angesetzt. Das lässt sich durchaus auf die Gesamtzahl der behandelten Patienten übertragen.

Jeder Arzt hat jedoch weiterhin einige Aspekte zu beachten: Erstens muss er die

Das Bundesverfassungsgericht vertraut darauf, dass Ärzte, die fachfremde Leistungen erbringen, ein entsprechendes Niveau gewährleisten.

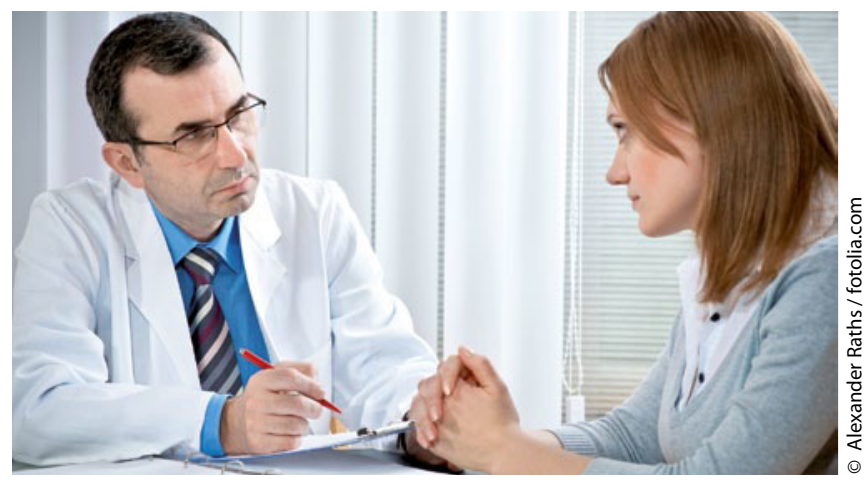

für ihn fachfremden Leistungen selbstverständlich beherrschen. Maßstab ist hierbei der jeweilige Facharztstandard. Bereits 1981 hat der Bundesgerichtshof (BGH) nämlich in einer arzthaftungsrechtlichen Entscheidung geurteilt, dass sich ein Arzt, der fachfremde Leistungen erbringt, nicht dadurch entlasten kann, dass er die fachspezifischen Veröffentlichungen und damit den Facharztstandard des fremden Gebietes nicht kannte.

Damals hatte ein Urologe ein Tuberkulostatikum verordnet, wobei pneumologischen Fachkreisen die Gefahr einer Schädigung des Sehnervs bekannt war, nicht aber dem Urologen. Der BGH führt hierzu - lapidar und richtig - aus, dass von jedem Arzt, der sich mit einer fachfremden Behandlung befasst, verlangt werden kann, dass er den hierbei zu beachtenden Standard kennt oder sich speziell darüber informiert.

\section{Haftpflichtschutz klären}

Zweitens sollte der Arzt seine Haftpflichtversicherung informieren und sich bestätigen lassen, dass für etwaige fach- fremde Behandlungen Versicherungsschutz besteht und es sich nicht um ein sogenanntes neues Risiko handelt, das vom Versicherungsschutz ausgenommen wäre.

Da die fachfremden Leistungen nur in geringfügigem Umfang erbracht werden, dürfen sie nicht als Tätigkeitsschwerpunkt beworben werden. Jedem Arzt ist bekanntlich die werbende Außendarstellung mit Tätigkeitsschwerpunkten erlaubt. Der Widerspruch zwischen der allein zulässigen geringfügigen fachfremden Tätigkeit und einem Tätigkeitsschwerpunkt wäre aber nicht aufzulösen.

Es ist zu erwarten, dass sich die berufsrechtlichen Probleme nach der Entscheidung des BVerG künftig eher in den Bereich der Kommunikation fachfremder Leistungen verlagern. Fachärzte sollten daher bei der Darstellung nicht zu marktschreierisch vorgehen. Berufsrechtliche Grenzen und vernünftige Außendarstellung lassen sich aber durchaus in Einklang bringen. RA Dr. Ingo Pflugmacher, Bonn 